\title{
Use of National Early Warning Score for observation for increased risk for clinical deterioration during post-ICU care at a surgical ward
}

This article was published in the following Dove Medical Press journal:

Therapeutics and Clinical Risk Management

\section{Pia Katrin Klepstad' \\ Trond Nordseth ${ }^{2,3}$ \\ Normunds Sikora ${ }^{4}$ \\ Pål Klepstad ${ }^{2,5}$}

'Faculty of Medicine, Riga Stradins University, Riga, Latvia; ' 2 epartment of Circulation and Medical Imaging, Faculty of Medicine and Health Sciences, Norwegian University of Science and Technology, Trondheim, Norway; ${ }^{3}$ Department of Emergency Medicine and Pre-hospital Services, St Olav University Hospital, Trondheim, Norway; ${ }^{4}$ Department of Surgery, Riga Stradins University, Riga, Latvia; ${ }^{5}$ Department of Anesthesiology and Intensive Care Medicine, St Olav University Hospital, Trondheim University Hospital, Trondheim, Norway
Correspondence: Pål Klepstad Department of Anesthesiology and Intensive Care Medicine, St Olav University Hospital, Trondheim University Hospital, Prinsesse Kristinas gt 3, 7006 Trondheim, Norway Tel +47 72575705

Email pal.klepstad@ntnu.no
Purpose: Patients transferred from an intensive care unit (ICU) to a general ward are at risk for clinical deterioration. The aim of the study was to determine if an increase in National Early Warning Score (NEWS) value predicted worse outcomes in surgical ward patients previously treated in the ICU.

Patients and methods: A retrospective observational study was conducted in a cohort of gastrointestinal surgery patients after transfer from an ICU/high dependency unit (HDU). NEWS values were collected throughout the ward admission. Clinical deterioration was defined by ICU readmission or death. The ability of NEWS to predict clinical deterioration was determined using a linear mixed effect model.

Results: We included 124 patients, age 65.9 $\pm 14.5,60 \%$ males with an ICU Simplified Acute Physiology Score II 33.8 \pm 12.7 . No patients died unexpectedly at the ward and 20 were readmitted to an ICU/HDU. The NEWS values increased by a mean of 0.15 points per hour (intercept 3.7, $P<0.001$ ) before ICU/HDU readmission according to the linear mixed effect model. NEWS at transfer from ICU was the only factor that predicted readmission (OR 1.32; 95\% CI 1.01-1.72; $P=0.04)$ at the time of admission to the ward.

Conclusion: Clinical deterioration of surgical patients was preceded by an increase in NEWS. Keywords: early warning score, post ICU patients, clinical deterioration, surgical

\section{Introduction}

Intensive care unit (ICU) patients are physiologically unstable patients, often with multi organ failure. ${ }^{1}$ Due to limited ICU resources with respect to staff and beds, patients are often discharged immediately after stabilization or even prematurely before full stabilization is achieved. ${ }^{2}$ Early or premature ICU discharge has the potential to increase readmission frequency, ${ }^{3}$ and increases mortality. ${ }^{4,5}$ Therefore, once discharged from an ICU or a high dependency unit (HDU), patients should be monitored closely by medical and nursing staff at the ward due to a significant risk of deterioration. ${ }^{6}$

Clinical deterioration is often preceded by abnormal physiology, ${ }^{7,8}$ and vital parameters should be monitored regularly. Several early warning scores (EWSs) with different designs have been developed to track variables such as blood pressure, pulse rate, respiration frequency, oxygen saturation, temperature and consciousness in hospitalized patients, ${ }^{9}$ none of which are specific for post ICU care. EWS values dictate the frequency and level of monitoring. ${ }^{10}$ Previous studies have shown that implementation of EWS reduce the incidence of serious adverse events in hospitalized patients. ${ }^{11,12}$ 
One of the EWSs in common use is the National Early Warning Score (NEWS) introduced in 2012 by the Royal College of Physicians. ${ }^{13}$ NEWS has been shown to have an improved ability to discriminate patients at risk of an adverse outcome,,$^{13}$ compared to 33 other EWSs. ${ }^{14}$ However, NEWS is not specifically developed or validated for post-ICU patients. A previous study by Uppanisakorn et a ${ }^{15}$ demonstrated that a high NEWS at ICU discharge predicted early clinical deterioration, but it is uncertain if NEWS can detect clinical deterioration after ICU discharge.

The aim of this study was to investigate the use of NEWS in post ICU patients treated at a gastrointestinal surgical ward. The primary research question was if deterioration in NEWS after transfer from the ICU for surgical patients predicted unexpected death or readmission to a higher level of care (ICU/HDU).

\section{Patients and methods Design}

This is a retrospective observational study of NEWS observations in a cohort of gastrointestinal surgical patients after transfer from an ICU/HDU. Patients were included between the dates of 01.01.16 and 30.06.17 at St Olav University Hospital.

\section{Setting}

St Olav University Hospital is a 983 bed, tertiary hospital located in Trondheim, Norway, serving about 720,000 inhabitants in the area of Mid-Norway. ${ }^{16}$ The ICU is an adult mixed case 10-bed unit. The decision for admittance to and discharge from the ICU and HDU is done on an individual basis by the intensivists and surgeons responsible for patient care. Surgical patients can be transferred from the ICU either directly to the ward, or first to a surgical HDU. The hospital has a gastrointestinal surgical department with two wards and a total of 48 beds. The hospital has no formal rapid response system except for a team responding to acute incidents such as cardiac or respiratory arrest.

\section{Patients}

The study screened all adult (age $\geq 18$ years) patients who were treated at the general ICU and transferred to the gastrointestinal surgical wards. Patients who had a stay in the HDU after ICU before admission to the ward were also included. Patients were identified using the ICU patient registry. For patients with several ICU admissions during the same hospitalization, only the first ICU admission followed by a ward admission was included. Patients with ICU outcomes of death, transferal to another ICU, specialty's ward or hospital, or a decision of withholding or withdrawing life-sustaining therapy were excluded.

\section{National Early Warning Score}

NEWS was introduced by the Royal College of Physicians with the aim to create a common system for detection of patients presenting with acute illness, or deteriorating patients at wards in the UK. ${ }^{13}$ It has later been adapted by numerous health care facilities, including St Olav University Hospital. ${ }^{17}$

NEWS values are obtained by nursing staff at the wards, and includes the following parameters: respiratory rate, oxygen saturation measured by pulse oximetry, supplementary oxygen, systolic arterial blood pressure, pulse rate, temperature and alertness. Observed values that deviate from defined normal values receive a score from 1 to 3 and are summed to a total score (Table 1).

The summed NEWS value is classified as low (score $0-4$ ), medium (score 5-6, or a single score of 3 in any parameter) or high (score $\geq 7$ ) clinical risk. The categories low, medium or high NEWS indicate the severity of the patient's condition, triggers a predefined action, and determines the frequency of measurements. Predefined actions determined by the Royal

Table I National Early Warning Score

\begin{tabular}{|c|c|c|c|c|c|c|c|}
\hline Physiological parameters & 3 & 2 & $\mathbf{I}$ & 0 & $\mathrm{I}$ & 2 & 3 \\
\hline Respiratory rate, $x / \min$ & $\leq 8$ & & $9-11$ & $12-20$ & & $21-24$ & $\geq 25$ \\
\hline Oxygen saturation, \% & $\leq 91$ & $92-93$ & $94-95$ & $\geq 96$ & & & \\
\hline Oxygen supplement & & Yes & & No & & & \\
\hline Temperature, ${ }^{\circ} \mathrm{C}$ & $\leq 35.5$ & & $35.1-36.0$ & $36.1-38.0$ & $38.1-39.0$ & $\geq 39.1$ & \\
\hline Systolic blood pressure, $\mathrm{mmHg}$ & $\leq 90$ & $91-100$ & $101-110$ & $111-219$ & & & $\geq 220$ \\
\hline Heart rate, $x / \min$ & $\leq 40$ & & $41-50$ & $51-90$ & $91-110$ & $111-130$ & $\geq|3|$ \\
\hline Level of consciousness, AVPU scale & & & & A & & & $\mathrm{V}, \mathrm{P}$ or $\mathrm{U}$ \\
\hline
\end{tabular}

Notes: Reprinted from Royal College of Physicians. National Early warning score (news): standardising the assessment of acute-illness severity in the NHS. Report of a working Party. London: RCP; 2012. (C) Royal College of Physicians 2012.13

Abbreviation: AVPU, alert, voice, pain, unresponsive. 
College of Physicians include assessment by a registered nurse (low risk group), urgent clinical review (medium risk group) and emergency clinical review (high risk group). NEWS values are measured at a time interval of $\leq 12$ hours (sum score 0 ), $\leq 6$ hours (low scores), $\leq 1$ hour (medium scores) and continuously (high scores).

NEWS was implemented as the EWS system used at the surgical wards at St Olav University Hospital in the spring of 2015, 1 year before this study was started.

\section{Data collection}

The following demographic variables were collected from the ICU registry: age at admission, gender, length of stay in the ICU, Simplified Acute Physiology Score (SAPS) II, ${ }^{18}$ use of respiratory support, continuous veno-venous hemodiafiltration, tracheostomy and ICU outcome. NEWS records calculated manually by the nursing staff, length of ward admission, main diagnosis and outcome (unexpected death, readmission ICU/HDU, discharged) after ward admission was retrieved from the patient's electronic journal (DocuLive, Siemens Nixdorf Information Systems, Oslo, Norway).

\section{Statistics and data analysis}

Statistical analyses were performed using the software IBM SPSS statistics version 22 and STATA version 15. The $R$ statistical software version 3.5.0 was used to generate the figures. ${ }^{19}$ Demographic data are shown using median (range) or mean (SD), as appropriate. The main outcome was death or readmission to ICU (yes/no). Patients were observed until they experienced the outcome or were discharged from the ward, whichever came first. Patient demographic characteristics were compared between those who experienced the main outcome or not using Student's $t$-test, Mann-Whitney U-test or chi-squared test, as appropriate. Variables that had a $P$-value of $<0.1$ were entered into a multivariate logistic regression with death/readmission as the dependent variable. A $P$-value $<0.05$ was considered to indicate statistical significance.

Plots of the changing NEWS value for individual patients with time are demonstrated in sequence plots generated by applying the R-package "TraMineR". ${ }^{20}$ The maximum NEWS value recorded in each 6-hour period was used in the sequence plots. The Locally Weighted Scatterplot Smoothing (LOWESS) plots of mean NEWS values were generated until the time point where death/readmission or discharge from ward was experienced in the two groups are demonstrated, respectively. To assess any possible differences in the change in NEWS between the two groups, a linear mixed effect model with varying slopes and intercepts was fit, taking interaction between time and group into account.

\section{Ethics}

The study was approved by the regional ethics committee and Data Protection Officer at St Olav University Hospital. The Data Protection Officer waived the need for an informed consent from each patient.

\section{Results}

Two hundred one individual patients were admitted 221 times to the ICU during the study period. Nineteen patients died in the ICU, 13 were transferred to the ward with a decision to withhold therapy, 41 were transferred to another ICU, specialty's ward or hospital, and 4 patients had no registered NEWS. This resulted in 124 patients included in the study (Figure 1).

The mean age was 65 years and $60 \%$ were male. The most frequent diagnoses were colorectal cancer, acute pancreatitis and ileus. The median ICU length of stay was 1.5 (range

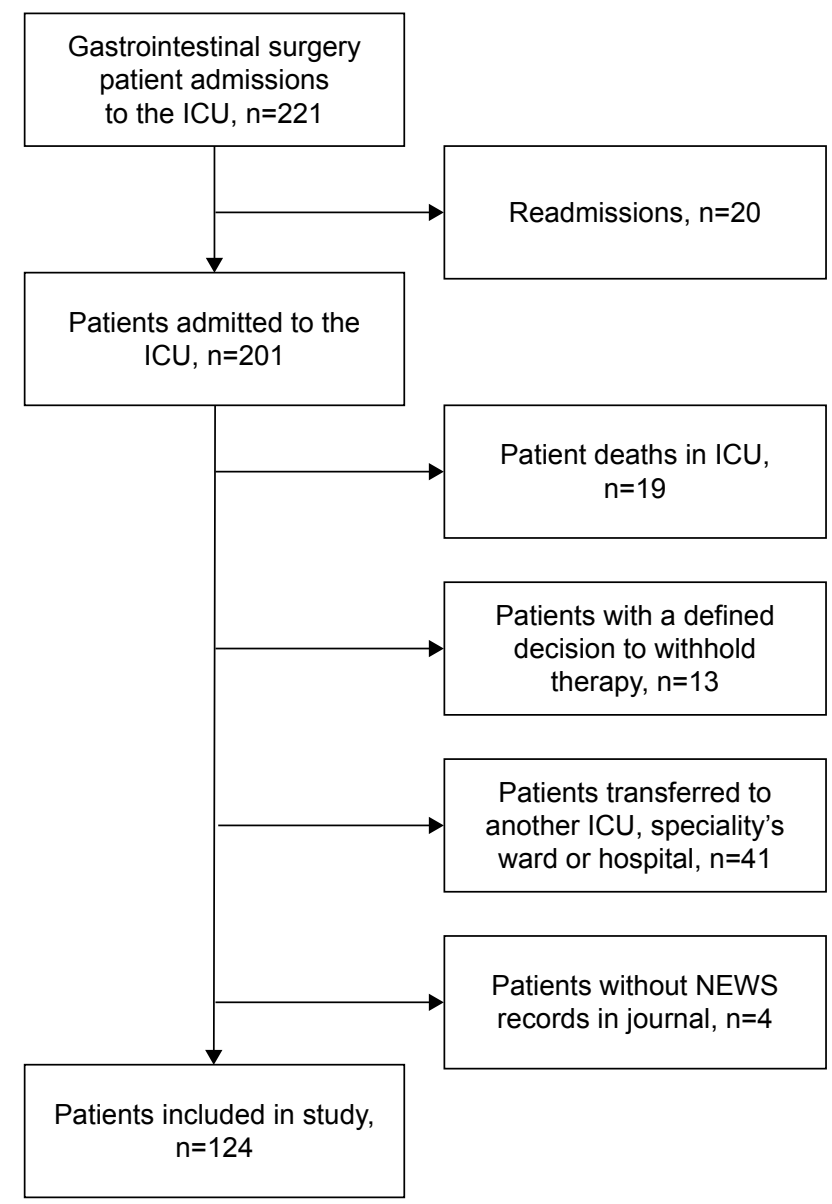

Figure I Flowchart shows patient exclusion process.

Abbreviations: ICU, intensive care unit; NEWS, National Early Warning Score. 
Table 2 Clinical characteristics and treatment details of 124 patients included in study

\begin{tabular}{|l|l|}
\hline Variables & Total (N=I24) \\
\hline Mean age (SD) & $65.9(14.5)$ \\
\hline Male gender (\%) & $74(59.9)$ \\
\hline Mean SAPSII (SD) & $33.8(12.7)$ \\
\hline Median length of stay ICU, days (min, max) & $1.5(0.04,30.8)$ \\
\hline Respiratory support (\%) & $63(50.8)$ \\
\hline Continuous veno-venous hemodiafiltration (\%) & $2(1.6)$ \\
\hline Tracheostomy (\%) & $4(3.2)$ \\
\hline Mean NEWS at ICU discharge (SD) & $2.7(1.9)$ \\
\hline Mean NEWS at outcome (SD) & $1.8(2.6)$ \\
\hline
\end{tabular}

Abbreviations: ICU, intensive care unit; NEWS, National Early Warning Score; SAPSII, Simplified Acute Physiology Score.

0.04-30.8) days. About half of the patients received respiratory support, and two patients needed continuous venovenous hemodiafiltration. Other demographic and treatment details are demonstrated in Table 2.

Twenty patients were readmitted to the ICU/HDU and eleven patients died at the ward. All patients who died at the ward did so after a decision of withdrawing therapy had been made. Thus, no patients met the criteria of unexpected death at the ward.

NEWS was observed between 0.08 and 54.9 days. A total of 4,132 NEWS values were included in the data set, corresponding to 3.7 NEWS values per treatment day, 5.4/day for the readmitted patients and 3.6/day for the discharged patients. Incomplete NEWS records were not included. On 283 occasions, NEWS records were missing for $>12$ hours.

Mean NEWS at start of registration at ward was 2.7 (SD 1.9), mean NEWS at the last observation was 1.8 (SD 2.6). For patients readmitted to the ICU/HDU the last observed NEWS value was significantly higher compared to the non-readmitted group; 6.0 (SD 3.2) vs 1.0 (SD 1.5) $(P<0.001)$, respectively (Table 3$)$. Also age, SAPSII score and first NEWS value observed at ward were associated with ICU/HDU readmission (Table 3).

In a logistic regression to evaluate the predictive effects of age, SAPSII score and first NEWS at ward on ICU/HDU readmission (Table 4), the first NEWS on the ward was the only significant parameter to predict ICU readmission (OR $1.32,95 \%$ CI 1.01-1.72, $P=0.04$ ) in the multivariate analysis. The regression analysis explained $14.6 \%$ (Nagelkerke $\mathrm{R}^{2}$ ) of the variability in the dataset.

The sequence of maximum NEWS at 6-hour intervals after transfer to the gastrointestinal surgical ward in readmitted and non-readmitted groups is demonstrated for individual patients in Figure 2. The figure illustrates that the readmitted patients seems to have a higher proportion of medium and high NEWS values, as illustrated by orange and red color. At 240 hours, all ICU/HDU readmissions observed in the study population had occurred. A number of patients in the non-readmitted group had transient increases in NEWS, but for those who the following NEWS improved we observed no need for readmission to the ICU/HDU.

Figure 3 is a LOWESS plot of the development of NEWS score for the last 180 hours before the ICU/HDU readmission or ward discharge was experienced for the two groups. The development in NEWS was different in the readmitted and non-readmitted groups. In the readmitted group, a marked increase in mean NEWS was observed 36 hours before outcome. In the non-readmitted group, a steady decline in mean NEWS was observed.

The results of the linear mixed effect model with respect to the development of mean NEWS value in the two groups is demonstrated in Table 5. There was a significant increase in

Table 3 Clinical characteristics of readmitted to ICU/HDU and non-readmitted groups

\begin{tabular}{|l|l|l|l|}
\hline Variables & Readmitted ICU/HDU (N=20) & Not readmitted (N=I 04) & P-value \\
\hline Mean age (SD) & $7 I .8(I I .2)$ & $64.8(I 4.8)$ & $0.044^{\mathrm{a}}$ \\
\hline Male gender (\%) & $\mathrm{I} 4(70.0)$ & $60(57.7)$ & $0.304^{\mathrm{b}}$ \\
\hline Mean SAPSII (SD) & $39.4(I 2.0)$ & $32.8(I 2.6)$ & $0.03 \mathrm{I}^{\mathrm{a}}$ \\
\hline Median length of stay ICU, days (min, max) & $2.33(0.04,24.4)$ & $\mathrm{I} .43(0.08,30.8)$ & $0.40 \mathrm{I}^{\mathrm{c}}$ \\
\hline Respiratory support (\%) & $\mathrm{I}(2(60.0)$ & $5 \mathrm{I}(49.0)$ & $0.369^{\mathrm{b}}$ \\
\hline Continuous veno-venous hemodiafiltration (\%) & $\mathrm{I}(5,0)$ & $\mathrm{I}(\mathrm{I}, 0)$ & $0.189^{\mathrm{b}}$ \\
\hline Tracheostomy (\%) & $\mathrm{I}(5,0)$ & $3(2,9)$ & $0.624^{\mathrm{b}}$ \\
\hline Mean NEWS at ICU discharge (SD) & $3.7(2.1)$ & $2.5(\mathrm{I})$ & $0.0 \mathrm{I}^{\mathrm{a}}$ \\
\hline Mean NEWS at outcome (SD) & $6.0(3.2)$ & $\mathrm{I} .0(\mathrm{I} .5)$ & $<0.00 \mathrm{I}^{\mathrm{a}}$ \\
\hline
\end{tabular}

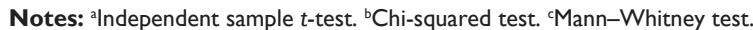

Abbreviations: HDU, high dependency unit; ICU, intensive care unit; NEWS, National Early Warning Score; SAPSII, Simplified Acute Physiology Score. 
Table 4 Multivariate logistic regression model of predictors associated with clinical deterioration and readmission to the ICU/ HDU

\begin{tabular}{|l|l|l|l|}
\hline Variables & OR & 95\% Cl & P-value \\
\hline Age & 1.03 & $0.98-1.07$ & 0.26 \\
\hline SAPSII & 1.03 & $0.99-1.07$ & 0.16 \\
\hline NEWS at ICU discharge & 1.32 & $1.01-1.72$ & 0.04 \\
\hline
\end{tabular}

Abbreviations: ICU, intensive care unit; NEWS, National Early Warning Score SAPSII, Simplified Acute Physiology Score.

NEWS score over time for readmitted patients (mean increase 0.15 points/hours) and they started at a higher mean score (1.11 points) as compared to patients who were not readmitted. Patients in the latter group demonstrated a significant decrease in mean NEWS over time, as can be seen in the LOWESS plots as well.

\section{Discussion}

We observed that readmission to the ICU/HDU for gastrointestinal surgical patients treated at the ward was preceded by an increase in NEWS value, while patients who were not readmitted to the ICU/HDU had a steady decrease in NEWS value over time. Furthermore, a higher NEWS at ICU/HDU discharge was the only predictive patient characteristic at ICU discharge of ICU/HDU readmission.

Several other studies confirm NEWS' ability to detect clinical deterioration by measurement of vital parameters within 24 hours of an adverse outcome, ie, transfer to the
ICU, cardiac arrest or death. ${ }^{14,21,22}$ This highlights the Royal College of Physicians proposed benefit of "identifying and responding to patients with unanticipated acute deterioration in their clinical condition whilst in hospital". ${ }^{13}$ However, none of these studies were done exclusively for surgical or post-ICU patients. The post-ICU patients may differ from other patients due to their preceding severe condition with threatening or confirmed organ failure, and usually a need for hemodynamic and respiratory support. This may make these patients particularly vulnerable after transfer to the ward, especially in cases of premature discharge before full stabilization is achieved. As a result of this, a number of patients are readmitted to the ICU, a risk that increases with unplanned discharges. ${ }^{23,24}$ The association with discharge NEWS and need for readmission in our and other studies suggest that some were discharged from the ICU/HDU with an incomplete resuscitation. ${ }^{15}$ Thus, high NEWS values at the time of ICU discharge could be a marker for a physiological state where transfer to the ward is inappropriate. Our results suggest that NEWS may also be at value for systematic use in order to adequately monitor the surgical post-ICU patient group at the ward, and to detect clinical deterioration in time to initiate appropriate interventions.

Several patients in our study had transient increases in NEWS, followed by normalization of NEWS and did not require readmission to the ICU. Transient changes in vital parameters causing an increased NEWS trigger a response, according to the severity classification given by the Royal

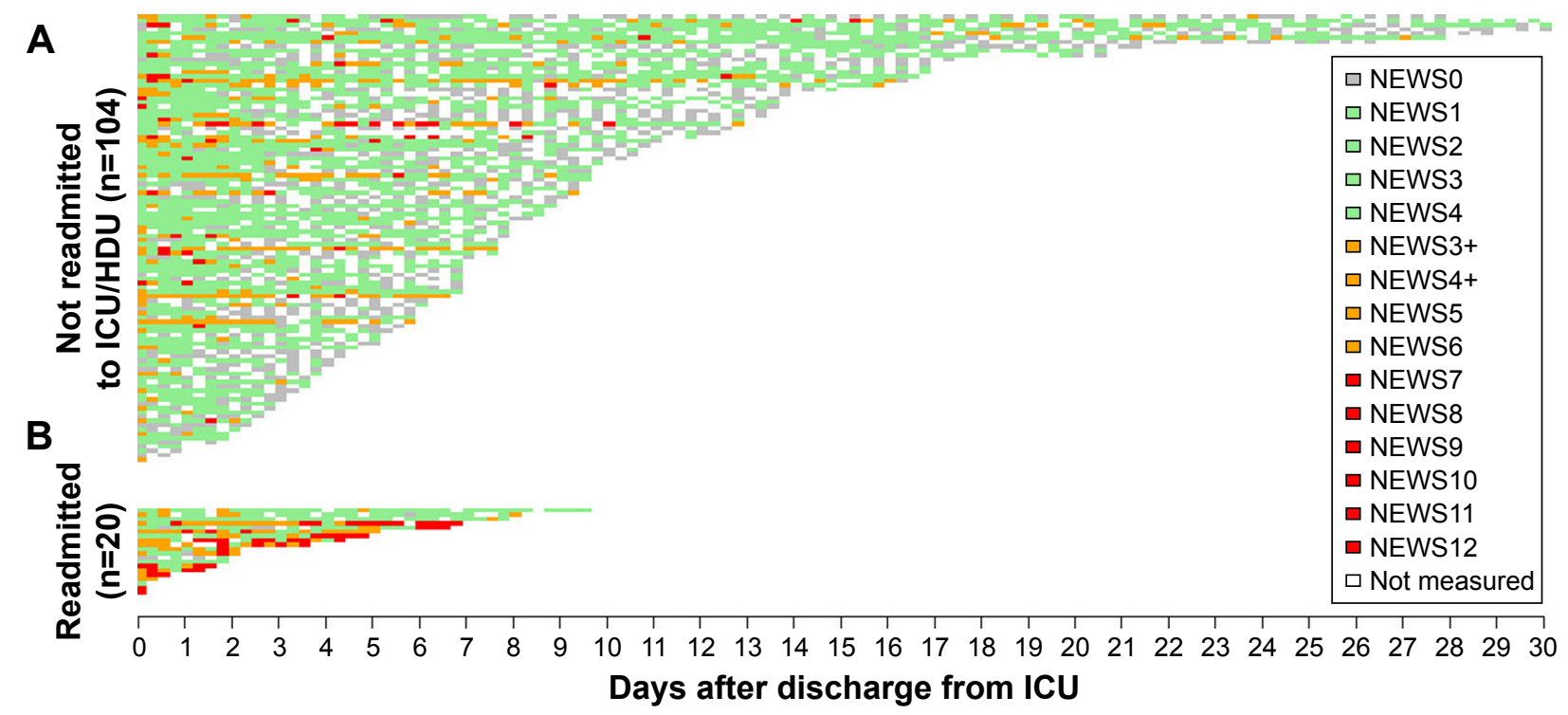

Figure 2 Sequence plot of maximum NEWS - first 30 days.

Notes: Patients not readmitted to the ICU/HDU are demonstrated in (A), and patients readmitted to the ICU/HDU are demonstrated in (B). There is one line per patient demonstrating the sequence of maximum NEWS score in each 6-hour period.

Abbreviations: HDU, high dependency unit; ICU, intensive care unit; NEWS, National Early Warning Score. 
A Hours before ICU readmission

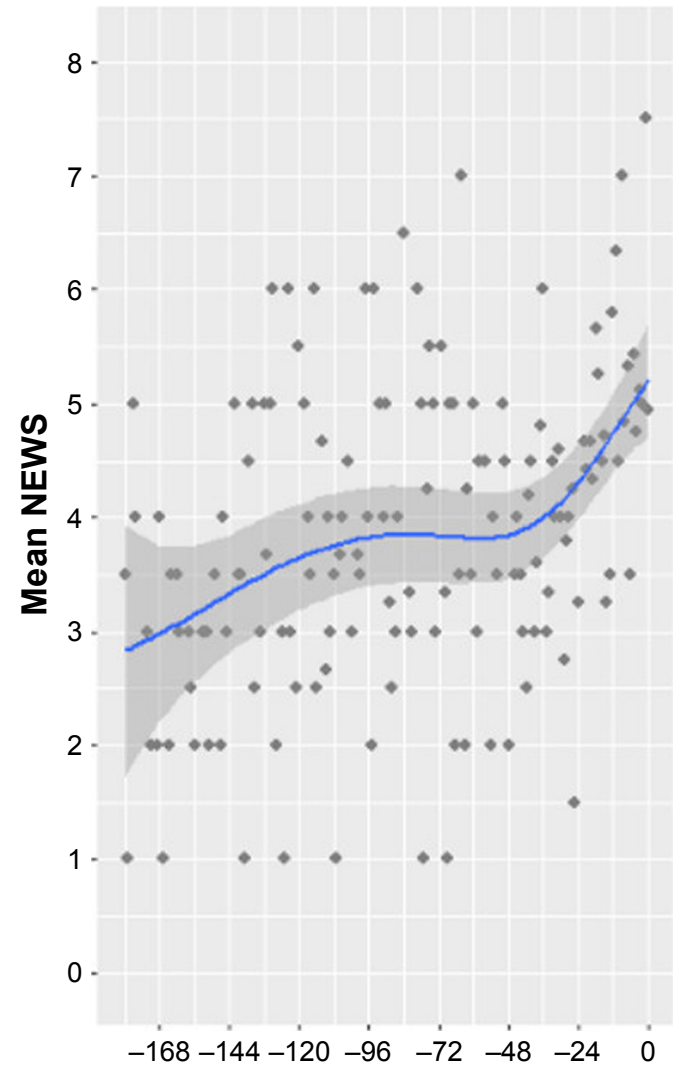

B Hours before discharge ward

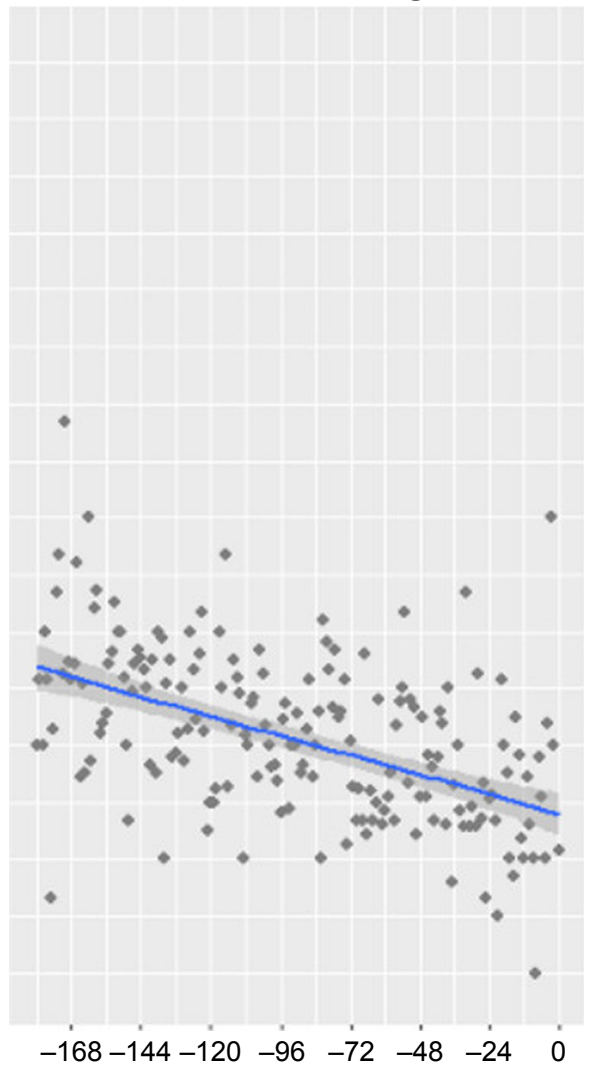

Figure 3 Locally Weighted Scatterplot Smoothing plot of the development of NEWS score for the last 180 hours before the ICU/HDU readmission or ward discharge was experienced.

Notes: (A) Patients readmitted to the ICU/HDU. (B) Patients not readmitted to the ICU/HDU.

Abbreviations: HDU, high dependency unit; ICU, intensive care unit; NEWS, National Early Warning Score.

College of Physicians, and actions may be taken at the ward to avoid unnecessary adverse events. Thus, the patients most at risk for ICU readmission are those where the initial increase in NEWS do not resolve either spontaneously or as a result of interventions performed by ward nurses and physicians.

NEWS is based upon measurements of vital parameters which represent only some of the follow up in the ward, and do not replace clinicians' and nurses' clinical experience and ability to interpret concerning symptoms such as chest pain or general discomfort. However, the use of a systematically obtained observation such as NEWS ensures that the patients' vital parameters are obtained at a predefined time interval and that all observations are documented. The collection of NEWS also ensures that the patients will not be

Table 5 Linear mixed effect model - change in mean NEWS score

\begin{tabular}{|c|c|c|c|c|}
\hline Parameters - fixed effects & Model parameter & Coef. & $95 \% \mathrm{Cl}$ & $P$-value \\
\hline Intercept baseline & $\beta_{0}$ & 2.59 & $(2.29-2.89)$ & $<0.001$ \\
\hline Time effect (hours) & $\beta_{1}$ & -0.009 & $(-0.01$ to -0.007$)$ & $<0.001$ \\
\hline Intercept - outcome group & $\beta_{2}$ & 1.11 & $(0.35-1.87)$ & 0.004 \\
\hline Interaction time (hours) vs group & $\beta_{3}$ & 0.024 & $(0.02-0.03)$ & $<0.001$ \\
\hline \multicolumn{5}{|l|}{ Parameters - random effects } \\
\hline Variance random intercepts & $b_{0,1}$ & 2.21 & $(1.67-2.93)$ & \\
\hline Variance random slopes & $b_{1, j}$ & 0.00008 & $(0.00006-0.00013)$ & \\
\hline Variance residual & $\varepsilon_{\mathrm{i}, \mathrm{j}}$ & 1.56 & $(1.48-1.64)$ & \\
\hline Covariance $\left(b_{0,1}, b_{1, j}\right)$ & & -0.0042 & $(-0.0079$ to -0.00053$)$ & \\
\hline
\end{tabular}

Notes: Model: $y_{i, j}=\beta_{0}+\beta_{1} \times$ time $+\beta_{2} \times$ group $+\beta_{3} \times$ time $\times$ group $+b_{0,1}+b_{1, j} \times$ time $+\varepsilon_{i, j}$. Restriced Maximum Likelihood (REML) was estimated. Abbreviations: Coef., coefficient; NEWS, National Early Warning Score. 
unattended by nurses for longer time intervals than between each NEWS measurement. Therefore, an inherent value of systematical screening is that the patients are observed by health care personnel which can add value by using their clinical experience in the evaluation of the patients. The importance of qualified health care personnel is also evident by the fact that the effects from detecting a clinical deterioration by deviating NEWS value is obviously dependent on the hospital competencies to further evaluate and treat the patients. The NEWS observation is just one part in a needed chain of events where detection must be followed by action. ${ }^{7}$

In our study, it seems that NEWS observations were adequately observed and responded to. Despite a number of patients who needed advanced care, we observed no unexpected deaths occurred at the wards. In this study, all deaths were preceded by a defined decision of withdrawing or withholding life-sustaining treatment. This suggests that in cases of observed deterioration in clinical condition, a physician was able to decide whether to start intensive care therapy or to withhold life-sustaining therapy. To be able to perform an end-of-life decision before an acute incident (ie, a cardiac arrest) benefits patients who avoid receiving futile and unpleasant treatment.

The minimum interval for NEWS records for each patient is 12 hours. Figure 3 illustrates that all except one patient had a NEWS of 3 or more 24 hours before readmitted to the ICU/HDU, suggesting that intervals of 12 hours or less will detect a clinically important deterioration. In our study, there were 283 intervals $>12$ hours between each complete NEWS registration, which composes $6.8 \%$ of the data set. Pedersen et $\mathrm{al}^{25}$ studied 2,835,331 NEWS records with a result of $10 \%$ incomplete NEWS records. In this study, $88 \%$ of the incomplete NEWS records were obtained within 12 hours of a complete record, which suggest that in many cases the time interval is as decided, but that the compliance in respect to obtain a complete NEWS assessment can be improved. To exclude one or more vital parameters may contribute to an underestimate of the patient's condition, and decrease the predictive capabilities of EWS. ${ }^{26,27}$ Both our and the study by Pedersen et al demonstrates that in clinical routine care some lack of compliance to observational protocols is to be expected.

We recognize that this study has some limitations. First, the data were obtained at a single center in two gastrointestinal surgical wards. Second, this study is observational and has no comparison with a patient cohort not observed by the use of NEWS. Third, we have no information about which intervention was performed for patients with a transient increase in NEWS. Fourth, due to the overall low number of patients included in the study, there is risk of overfitting the applied regression models which may limit the generalizability of the findings. Finally, this retrospective study relies on compliance by health care workers to record NEWS correctly. Human factors such as calculation errors, ${ }^{28}$ incorrect recorded time of measurements and biases toward values within the limits for producing a zero score may have occurred. ${ }^{25}$

\section{Conclusion}

In conclusion, we observed that deterioration of surgical patients transferred to a ward was preceded by an increase in NEWS value. Thus, this study suggests that monitoring with NEWS at the surgical ward is of value after ICU discharge.

\section{Acknowledgments}

We appreciate the support from physicians and nurses working at the gastrointestinal surgical wards at St Olav University Hospital.

\section{Disclosure}

The authors report no conflicts of interest in this work.

\section{References}

1. Ræder J, Flaatten H. Intensivmedisin. Anestesiologi en innføringsbok. Oslo: Gyldendal akademisk; 2016:119-157. Norwegian.

2. Chrusch CA, Olafson KP, McMillan PM, Roberts DE, Gray PR. High occupancy increases the risk of early death or readmission after transfer from intensive care. Crit Care Med. 2009;37(10):2753-2758.

3. Utzolino S, Kaffarnik M, Keck T, Berlet M, Hopt UT. Unplanned discharges from a surgical intensive care unit: readmissions and mortality. J Crit Care. 2010;25(3):375-381.

4. Kramer AA, Higgins TL, Zimmerman JE. Intensive care unit readmissions in U.S. hospitals: patient characteristics, risk factors, and outcomes. Crit Care Med. 2012;40(1):3-10.

5. Nguyen MC, Strosberg DS, Jones TS, et al. Mortality and readmission of outcomes after discharge from the surgical intensive care unit to long-term, acute-care hospitals. Surgery. 2017;161(5):1367-1375.

6. Kaben A, Corrêa F, Reinhart K, et al. Readmission to a surgical intensive care unit: incidence, outcome and risk factors. Crit Care. 2008; 12(5):R123.

7. Smith GB. In-hospital cardiac arrest: is it time for an in-hospital 'chain of prevention'? Resuscitation. 2010;81(9):1209-1211.

8. Kause J, Smith G, Prytherch D, et al. A comparison of antecedents to cardiac arrests, deaths and emergency intensive care admissions in Australia and New Zealand, and the United Kingdom - the ACADEMIA study. Resuscitation. 2004;62(3):275-282.

9. Gao H, McDonnell A, Harrison DA, et al. Systematic review and evaluation of physiological track and trigger warning systems for identifying at-risk patients on the ward. Intensive Care Med. 2007;33(4):667-679.

10. Smith GB, Prytherch DR, Meredith P, Schmidt PE. Early warning scores: unravelling detection and escalation. Int $J$ Health Care Qual Assur. 2015;28(8):872-875.

11. Paterson R, MacLeod D, Thetford D, et al. Prediction of in-hospital mortality and length of stay using an early warning scoring system: clinical audit. Clin Med. 2006;6(3):281-284. 
12. Moon A, Cosgrove JF, Lea D, Fairs A, Cressey DM. An eight year audit before and after the introduction of modified early warning score (MEWS) charts, of patients admitted to a tertiary referral intensive care unit after CPR. Resuscitation. 2011;82(2):150-154.

13. Royal College of Physicians. National Early Warning Score (NEWS): standardising the assessment of acute-illness severity in the NHS. Report of a Working Party. London: RCP; 2012.

14. Smith GB, Prytherch DR, Meredith P, Schmidt PE, Featherstone PI. The ability of the National Early Warning Score (NEWS) to discriminate patients at risk of early cardiac arrest, unanticipated intensive care unit admission, and death. Resuscitation. 2013;84(4):465-470.

15. Uppanisakorn S, Bhurayanontachai R, Boonyarat J, Kaewpradit J. National Early Warning Score (NEWS) at ICU discharge can predict early clinical deterioration after ICU transfer. J Crit Care. 2018;43:225-229.

16. Stolav.no [homepage on the Internet]. St. Olav University Hospital: Key figures from 2016. [updated June 19, 2017; cited May 05, 2017 ]. Available from: https://stolav.no/en/about-the-hospital/key-figuresfrom-2016. Assessed July 26, 2018.

17. Stolav.no [homepage on the Internet]. Prosjektrapport: Systematisk måling av vitale parametere på sengeposter på St. Olavs Hospital. Available from: https://stolav.no/seksjon-avdeling/Documents/Prosjektrapport_Måling\%20av\%20vitalparametere\%20på\%20sengepost $\% 20$ StOlav_versjon\%202.0.pdf. Assessed February 16, 2019.

18. Le Gall JR, Lemeshow S, Saulnier F. A new simplified Acute Physiology score (saps II) based on a European/North American multicenter study. JAMA. 1993;270(24):2957-2963.

19. R Core Team. R: A Language and Environment for Statistical Computing. R foundation for statistical computing; 2016. Available from: http://www.r-project.org/. Assessed February 16, 2019.

20. Gabadinho A, Ritschard G, Müller NS, Studer M. Analyzing and visualizing state sequences in R with TraMineR. J Stat Soft. 2011;40(4):1-37.
21. Green M, Lander H, Snyder A, Hudson P, Churpek M, Edelson D. Comparison of the between the flags calling criteria to the MEWS, news and the electronic cardiac arrest risk triage (eCART) score for the identification of deteriorating ward patients. Resuscitation. 2018;123:86-91.

22. Smith GB, Prytherch DR, Jarvis S, et al. A comparison of the ability of the physiologic components of medical emergency team criteria and the U.K. National Early Warning Score to discriminate patients at risk of a range of adverse clinical outcomes. Crit Care Med. 2016;44(12): 2171-2181.

23. Vollam S, Dutton S, Lamb S, Petrinic T, Young JD, Watkinson P. Out-of-hours discharge from intensive care, in-hospital mortality and intensive care readmission rates: a systematic review and meta-analysis. Intensive Care Med. 2018;44(7):1115-1129.

24. Ponzoni CR, Corrêa TD, Filho RR, et al. Readmission to the intensive care unit: incidence, risk factors, resource use, and outcomes. A retrospective cohort study. Ann Am Thorac Soc. 2017;14(8):1312-1319.

25. Pedersen NE, Rasmussen LS, Petersen JA, Gerds TA, Østergaard D, Lippert A. A critical assessment of early warning score records in 168,000 patients. J Clin Monit Comput. 2018;32(1):109-116.

26. Ludikhuize J, Smorenburg SM, de Rooij SE, de Jonge E. Identification of deteriorating patients on general wards; measurement of vital parameters and potential effectiveness of the modified early warning score. J Crit Care. 2012;27(4):424.e7-e13.

27. Clifton DA, Clifton L, Sandu DM, et al. "Errors" and omissions in paper-based early warning scores: the association with changes in vital signs - a database analysis. BMJ Open. 2015;5(7):e007376.

28. Kolic I, Crane S, McCartney S, Perkins Z, Taylor A. Factors affecting response to National Early Warning Score (NEWS). Resuscitation. 2015;90:85-90.
Therapeutics and Clinical Risk Management

\section{Publish your work in this journal}

Therapeutics and Clinical Risk Management is an international, peerreviewed journal of clinical therapeutics and risk management, focusing on concise rapid reporting of clinical studies in all therapeutic areas outcomes, safety, and programs for the effective, safe, and sustained use of medicines. This journal is indexed on PubMed Central, CAS,

\section{Dovepress}

EMBase, Scopus and the Elsevier Bibliographic databases. The manuscript management system is completely online and includes a very quick and fair peer-review system, which is all easy to use. Visit http://www.dovepress.com/testimonials.php to read real quotes from published authors. 